\title{
New preconditioning strategy for Jacobian-free solvers for variably saturated flows with Richards' equation
}

\author{
Daniil Svyatskiya, ${ }^{\mathrm{a}, *}$, Konstantin Lipnikov $^{\mathrm{a}, 2}$, David Moulton $^{\mathrm{a}, 3}$ \\ ${ }^{a}$ Los Alamos National Laboratory, Los Alamos, NM 87545, USA
}

\begin{abstract}
We develop a new approach for solving the nonlinear Richards' equation arising in variably saturated flow modeling. The growing complexity of geometric models for simulation of subsurface flows leads to the necessity of using unstructured meshes and advanced discretization methods. Typically, a numerical solution is obtained by first discretizing PDEs and then solving the resulting system of nonlinear discrete equations with a NewtonRaphson-type method. Efficiency and robustness of the existing solvers rely on many factors, including an empiric quality control of intermediate iterates, complexity of the employed discretization method and a customized preconditioner. We propose and analyze a new preconditioning strategy that is based on a stable discretization of the continuum Jacobian. We will show with numerical experiments for challenging problems in subsurface hydrology that this new preconditioner improves convergence of the existing Jacobian-free solvers 3-20 times. We also show that the Picard method with this preconditioner becomes a more efficient nonlinear solver than a few widely used Jacobian-free solvers.
\end{abstract}

Keywords: Richards' equation, Variable saturated flows, Nonlinear solver, Picards' method

\section{Introduction}

Variably saturated flow in porous media is a critical process in a wide variety of environmental applications, including contaminant transport in the subsurface and carbon cycling in degrading permafrost. In these applications, Richards' equation [33] is often used to model variably saturated subsurface flow. Typically, this equation describes the movement of the liquid phase in a two-phase system under the simplifying assumption that the movement of the gas phase can be neglected. The flexibility and appeal of this approximation is evident in the generalization of Richards' formulation to more complex settings, such as non-isothermal three-phase (ice, water, air) systems (e.g., modeling hydrology in permafrost) [20]. However, this simplification also makes Richards' equation a strongly nonlinear parabolic partial differential equation (PDE), creating significant challenges for accurate discretization schemes and efficient iterative solvers.

${ }^{*}$ Corresponding author

${ }^{1}$ Email: dasvyat@lanl.gov

${ }^{2}$ Email: lipnikov@lanl.gov

${ }^{3}$ Email: moulton@lanl.gov 
Early work on numerical methods for Richards' equation showed that the low-order implicit discretization in time of its mixed-form, in concert with a finite volume discretization in space, conserves mass [9]. Although, it was later shown that good mass conservation could be obtained with the pressure-head form of Richards' equation by using higher-order time integration strategies [35, 28], the mixed-form has remained the most popular. In addition, it was found that using the flow direction to guide upwinding of the relative permeability is necessary to capture its strong nonlinearity, particularly with infiltration into dry soil (cf. [3]). This early research focused on orthogonal cartesian grids with isotropic or grid-aligned anisotropic permeabilities (K-orthogonal), for which the two-point flux approximation (TPFA) of the Darcy flux is accurate. Moreover, in this setting other important properties of the continuum model are naturally preserved in the discretization (e.g., symmetry, discrete maximum principle), which helps support the development of robust non-linear solvers. Although, a wide range of non-linear solvers have been investigated, Newton-based methods are the most popular for the TPFA discretization. Typically, the Jacobian matrix is computed by differentiating the discrete nonlinear system of equations analytically with respect to the discrete pressure unknowns. This discrete Jacobian matrix is non-symmetric but positive definite, and for certain upwinding formulations, naturally picks up the appropriate upwinding needed for stability of the first-order derivatives. However, complexity and cost do increase if complex models of porosity or density are incorporated, particularly if these models require interpolation of tabular data.

Compounding these challenges is the geometric complexity of the subsurface environment, which includes features such as sloping topography along with dipping and pinchedout stratigraphy. Often, unstructured grids are used to capture these complex features without stair stepping material interfaces and topography. Unfortunately, the popular TPFA approximation of the Darcy flux remains accurate only for K-orthogonal grids, and in many complex geometries these grids cannot be constructed. Using the TPFA discretization on general grids without the K-orthogonal property, such as grids capturing a layer pinch-out, introduces significant errors with clear grid-based artifacts [23]. Consequently, in recent years there has been significant research on more advanced discretizations such as the Multi-point Flux-Approximation (MPFA) [40], Mixed Finite Element (MFE) [4], Mimetic Finite Difference (MFD) [17], Hybrid Finite Volume (HFV), and Mixed Finite Volume (MFV) methods [14].

These advanced discretization methods offer accurate schemes for more general element shapes, which eases the challenge of mesh generation for complex geometries. However, these methods add significant complexity to the discretization and solver. For example, edge-based (in two dimensions) or face-based (in three dimensions) pressure unknowns are added in the MFD discretization; severe mesh distortion or misalignment with the permeability may destroy the discrete maximum principle; and existing approaches to upwinding the relative permeability breaks the symmetry of the discretization itself. In addition, these methods often generate a parameterized family of accurate discretizations, creating the opportunity for optimization to restore these important properties of the continuum model. Although, this complexity in the discretization is manageable, it is problematic for the direct analytic discretization of the nonlinear discrete system, as it is costly or overly complex to evaluate and the resulting Jacobian matrix may be singular despite the use of upwinding for the relative permeability. Shifting to nonlinear discretizations, such as 
the nonlinear finite volume method [25, 26], alleviates some of these issues and provides the only positivity preserving schemes on general polyhedral grids. But in this case, there is no closed form expression for the discretization, so computation of an analytic discrete Jacobian is not possible.

Thus, the additional complexity associated with advanced discretizations, as well as the cost of computing the Jacobian, has created significant interest in Jacobian-free and inexact Newton methods. Unfortunately, the strong nonlinearity in the relative permeability limits the efficacy of typical simplifications, such as freezing the Jacobian matrix during the solve, or freezing coefficients in the nonlinear operator to form the Picard preconditioner [19]. Methods such as the Jacobian-free Newton-Krylov method [21, 22] only need the action of the Jacobian matrix on a vector, which is approximated through the numerical computation of the Gâteaux derivative. However, in practice the accompanying Krylov method requires a robust and scalable preconditioner, and hence, the challenge of approximating the Jacobian matrix is central to these methods. Quasi-Newton methods such as Broyden [5, 21] and BFGS ([30], Sec.8.1), as well as accelerated Jacobian-free fixed-point methods such as Anderson mixing [2] and Nonlinear Krylov Acceleration (NKA) [7,6] also have a critical need for a preconditioner that approximates the Jacobian well. Thus, our goal is to develop a new Jacobian-free nonlinear solver that builds a robust and scalable preconditioner as part of its design.

The underlying idea in this work is to reverse the order of the discretization and linearization steps in the development of the nonlinear solver. Specifically, by performing the linearization step first, the analytic Jacobian of the continuum model is derived and then analyzed to establish requirements for accurate and stable discretizations. Hence, this strategy allows us to take full advantage of advanced discretization methods, including optimization strategies. This approach was used successfully for non-equilibrium radiationdiffusion on quadrilateral grids [41]. Here Richards' equation presents additional challenges, including the presence of gravity and its interaction with the upwinding of the relative permeability. In addition, it enables the use of physical reasoning and mathematical analysis to motivate effective approximations of the analytic Jacobian. This type of analysis was used to develop a symmetric preconditioner for the Jacobian of Richards' equation [19], and has been used in the design of semi-implicit physics-based preconditioners for JFNK solvers in plasma physics applications, such as the simulation of Hall magnetohydrodynamics [11].

To develop the new Jacobian-free solver we begin with the linearization of Richards' equation and derive the linear partial differential operator that is the analytic Jacobian. We analyze the terms in this operator, and develop conditions under which the linearized operator is positive definite. This analysis underpins the advanced discretization of this linear operator, ensuring that it is both accurate and conditionally positive definite. These properties ensure that the corresponding linear system is amenable to efficient scalable iterative solvers, such as algebraic multigrid. In addition, it ensures that this linear operator is an accurate approximation of the Jacobian in the sense that asymptotically as the mesh size tends to zero, the Jacobian of the discrete nonlinear system and the discretization of the analytical Jacobian (linearized operator) converge. Thus, we refer to this linearized operator as a quasi-optimal preconditioner. Using this preconditioner in the context of the Picard iteration leads to the new nonlinear solver. An interesting feature of this new solver is that it can be expressed in a way that connects the properties of the discretization to 
the properties of each iterate. Thus, creating interesting opportunities for future research in controlling physical constraints such as positivity over the entire sequence of iterates. To demonstrate the efficacy of this new solver we consider the challenging problem of water infiltration into distinct layers of dry soil, and compare the performance of the new preconditioner for three popular Jacobian-free nonlinear solvers, JFNK, NKA, and Picard.

The paper outline is as follows. In Section 2 we introduce Richards' equation in mixedform and highlight key steps in discretizing it with the MFD method. Nonlinear solvers are introduced in Section 3, with the Picard method providing the framework for both inexactNewton methods and accelerated methods (e.g, NKA). In addition, the new nonlinear solver arising through the use of the new preconditioner in a Picard iteration is presented in Section 3.3. For completeness, key algorithmic features of the JFNK solver are highlighted in Section 3.4. In Section 4, numerical results for two challenging problems of infiltration into layers of dry soil are used to compare the impact of the new preconditioner on the performance of these nonlinear solvers.

\section{Richards equation and its discretization}

Let $\Omega$ be a two-dimensional polygonal domain with the Lipschitz boundary $\Gamma$. The Richards equation for an unknown pressure $p$ written in a mixed form reads:

$$
\frac{\partial(\rho \phi s)}{\partial t}+\operatorname{div}(\rho \mathbf{q})=0, \quad \mathbf{q}=-\mathbf{K} \frac{k(s)}{\mu}(\nabla p-\rho \mathbf{g}) .
$$

where $\mathbf{q}\left[\mathrm{m} \cdot \mathrm{s}^{-1}\right]$ is the Darcy velocity, $\mathbf{g}$ is the gravity vector, and $s\left(p_{c}\right)[-]$ is the water saturation function of capillary pressure $p_{c}=p_{a t m}-p[\mathrm{~Pa}]$. The first equation represents the mass balance equation and the second one is the nonlinear Darcy law. The medium properties are described by porosity $\phi[-]$, absolute permeability $\mathbf{K}\left[\mathrm{m}^{2}\right]$ and relative permeability $k(s)[-], 0<k(s) \leq 1$. The fluid properties are described by density $\rho$ $\left[\mathrm{kg} \cdot \mathrm{m}^{-3}\right]$ and viscosity $\mu[\mathrm{Pa} \cdot \mathrm{s}]$.

In this study, we consider constant $\rho$ and $\mu$. We assume that $\Omega$ consists of a few different soils $\Omega_{s}$ characterized by full constant symmetric positive definite tensors $\mathbf{K}_{s}$, porosity $\phi_{s}$ and different water retention models. We assume that $\phi_{s}$ is constant inside each soil. A water retention model establishes the relationship between pressure and saturation functions, $s(p)$, and provides a closed form analytical expression for the relative permeability as a function of pressure, $k(p)=k(s(p))$. Typically, the relative permeability is a non-decreasing function of $p$ :

$$
\frac{\partial k}{\partial p} \geq 0
$$

In this paper we consider the relative permeability model defined by van Genuchten and Mualem equations, see [36]:

$$
k_{r}=\sqrt{s}\left[1-\left(1-s^{1 / m}\right)^{m}\right]^{2}, \quad s=\left[1+\left(\alpha p_{c}\right)^{n}\right]^{-m},
$$

where $m, n=\frac{1}{1-m}$ and $\alpha$ are parameters of the water retention model.

An important mathematical property of Richards' equation is that the steady-state solution of (1) satisfies the strong form of the maximum principle inside each soil provided 
that $\mathbf{K}_{s}$ is constant [29]. Since, the maximum principle holds in a vicinity of the steadystate solution, a numerical scheme that respects it will lead to a more accurate simulation, especially when the solution dynamics is smooth.

The fluid flow is driven by boundary conditions. We split the boundary $\Gamma$ into two pieces $\Gamma_{D}$ and $\Gamma_{N}$ and set

$$
\begin{aligned}
p & =g_{D}(\mathbf{x}) \quad \text { on } \Gamma_{D} \\
\mathbf{q} \cdot \mathbf{n} & =g_{N}(\mathbf{x}) \quad \text { on } \Gamma_{N} .
\end{aligned}
$$

Then the system (1)-(4) is closed by initial conditions for a pressure solution. In many practical simulations the initial pressure profile is unknown therefore it has to be computed in a preliminary stage which is referred to as a steady-state calculation.

\subsection{Mimetic finite difference method}

Numerical simulations on unstructured meshes require advanced discretization methods. We discretize the system of equations (1) using the mimetic finite difference (MFD) method. This method allows us to use arbitrary shaped polygons (if necessary, non-convex and degenerate polygons) and polyhedra (in 3D) to model complex subsurface stratigraphy and surface topography.

Let us consider a conformal partition of the computational domain $\Omega$ into polygonal cells $E$. Let $|E|$ denote the area of polygonal cell $E,|f|$ denote the length of mesh edge $f$, and $\mathbf{n}_{f}$ denote the unit normal vector to $f$ fixed once and for all.

The MFD method uses two types of pressure unknowns: cell-based (one unknown, $p_{E}$, per mesh cell) and edge-based (one unknown, $\lambda_{f}$, per mesh edge). The discrete velocity unknowns are only edge-based (one unknown, $q_{f}$, per mesh edge that represents the average flux $\mathbf{q} \cdot \mathbf{n}_{f}$ across edge $f$ ).

Let $\mathbf{q}_{E}$ be the vector of flux unknowns in cell E. Integrating the mass balance equation of (1) over cell $E$, replacing the integrals by central-point quadratures, applying the implicit backward-Euler time discretization, and dividing by $|E|$ we obtain

$$
\rho \phi_{E} \frac{s\left(p_{E}\right)-s\left(p_{E, 0}\right)}{\Delta t}+\operatorname{div}_{E}^{h}\left(\rho \mathbf{q}_{E}\right)=\rho \phi_{E} \frac{s\left(p_{E}\right)-s\left(p_{E, 0}\right)}{\Delta t}+\frac{1}{|E|} \sum_{f \in \partial E} \rho \xi_{E, f} q_{f}|f|=0
$$

where $p_{E, 0}$ is cell-based pressure unknown at the previous time step and $\xi_{E, f}$ is either 1 or -1 depending on the orientation of the fixed normal $\mathbf{n}_{f}$. Let $A_{E}=\left(\rho \phi_{E}\right) / \Delta t$. The algebraic form of (5) is

$$
A_{E} s\left(p_{E}\right)+\frac{\rho}{|E|} B_{E} \mathbf{q}_{E}=A_{E} s\left(p_{E, 0}\right)
$$

with the obvious definition of the entries in the row matrix $B_{E}$.

To discretize the constitutive equation in (1), we introduce value $k_{f}$ that approximates the relative permeability on edge $f$. The value $k_{f}$ is computed using the upwind approach based on the sign of flux $\mathbf{q}_{f}$ and orientation of the fixed normal $\mathbf{n}_{f}$. Let edge $f$ be shared by cells $E_{i}$ and $E_{j}$. Then, $k_{f}=k\left(p_{E_{i}}\right)$ if flow moves from cell $E_{i}$ to cell $E_{j}$; otherwise, $k_{f}=k\left(p_{E_{j}}\right)$. Let $K_{E}$ be the cell-based diagonal matrix with values $k_{f}$ on the diagonal and the dimension equal to the number of edges. In general, various flow regimes (infiltration, drainage, capillary rise) may apply different approaches to compute values $k_{f}$ [37]. 
Let $\lambda_{E}$ be the vector of edge-based pressure unknowns in a cell $E$. To approximate the gravity term, we introduce a cell-based vector $\mathbf{g}_{E}$, whose entries are $\frac{\rho}{\mu} \mathbf{K g} \cdot \mathbf{n}_{f}$, where $f \in \partial E$. Then the discrete form of the constitutive equation in cell $E$ is given by

$$
\mathbf{q}_{E}=K_{E} W_{E}\left(B_{E}^{T} p_{E}-C_{E} \lambda_{E}\right)+K_{E} \mathbf{g}_{E} .
$$

where $C_{E}$ is the cell-based diagonal matrix formed by the entries of matrix $B_{E}^{T}$, and $W_{E}$ is a symmetric positive definite matrix (the inverse of the mass matrix). The derivation of $W_{E}$ captures the influence of the cell geometry as well as the orientation of the absolute permeability tensor. The explicit formula for $W_{E}$ is at the heart of the MFD method. The detailed derivation of matrix $W_{E}$ can be found in [17]. When the mesh is orthogonal and the absolute permeability tensor is diagonal (with primary directions aligned with the mesh axes), this matrix is diagonal. In the case of a fully saturated soil, the discrete constitutive equation is exact for any linear pressure function.

The system of cell-based balance and constitutive equations is closed by the normal flux continuity constraint and boundary conditions. The pressure boundary conditions provide exact values for $\lambda_{f}$. The Neumann boundary conditions specify values of $\mathbf{q}_{f}$.

\subsection{Darcy flux in one dimension}

In one dimension, formula (6) leads to the conventional finite difference formula. Indeed, consider two neighboring cells $E_{1}$ and $E_{2}$ with the interface $f$ such that $\mathbf{n}_{f}=-1$ and

$\mathbf{g}=-|g|$. The matrices $W_{E_{i}}$ become diagonal matrices with values $2 \frac{\mathbf{K}_{E_{i}}}{\mu h_{E_{i}}}, i=1,2$, on the diagonal. Formula (6) gives two one-sides fluxes:

$$
q_{f}^{(1)}=\frac{k_{f}}{\mu} \mathbf{K}_{E_{1}}\left(\frac{p_{E_{1}}-\lambda_{f}}{h_{E_{1}} / 2}+\rho|g|\right) \quad \text { and } \quad q_{f}^{(2)}=\frac{k_{f}}{\mu} \mathbf{K}_{E_{2}}\left(\frac{\lambda_{f}-p_{E_{2}}}{h_{E_{2}} / 2}+\rho|g|\right) .
$$

The flux continuity allows us to elliminate $\lambda_{f}$ obtaining

$$
q_{f}^{(1)}=q_{f}^{(2)}=\frac{k_{f}}{\mu}\left(h_{E_{1}}+h_{E_{2}}\right)\left(\frac{h_{E_{1}}}{2 \mathbf{K}_{E_{1}}}+\frac{h_{E_{2}}}{2 \mathbf{K}_{E_{2}}}\right)^{-1}\left(\frac{p_{E_{1}}-p_{E_{2}}}{\left(h_{E_{1}}+h_{E_{2}}\right) / 2}+\rho|g|\right) .
$$

This formula uses the expected harmonic averaging of absolute permeabilities. If $k_{f}$ is defined by the first-order upwind algorithm, the resulting finite difference scheme is the first-order accurate in space. The MFD scheme preserves this order of accuracy on general meshes.

\section{Nonlinear solvers}

Let us rewrite Richards' equation in the form $\mathcal{F}(p)=0$ and consider one time step of size $\Delta t$. Applying the backward Euler discretization in time, the MFD discretization method in space, and eliminating fluxes, we obtain a discrete system of nonlinear equations $\mathcal{F}_{h}\left(p_{h}\right)=0$, where $p_{h}$ is the vector of all degrees of freedom for pressure (i.e. $p_{E}$ and $\lambda_{E}$ ). The functional $\mathcal{F}_{h}$ can be expressed as a sum of three terms:

$$
\mathcal{F}_{h}\left(p_{h}\right)=\mathcal{A}_{h}\left(p_{h}\right)+\mathcal{D}_{h}\left(p_{h}\right) p_{h}+\mathcal{B}_{h}\left(p_{h}\right)
$$


where $\mathcal{A}_{h}\left(p_{h}\right)$ is the accumulation term, $\mathcal{D}_{h}\left(p_{h}\right)$ is the matrix operator representing the diffusion term $\operatorname{div}(\rho \mathbf{q})$, and $\mathcal{B}_{h}$ is the discrete functional representing the contributions of the boundary conditions and source terms.

Let $\mathcal{J}_{h}\left(p_{h}\right)$ denote the Jacobian matrix (or, simply, Jacobian) of $\mathcal{F}_{h}$ evaluated on vector $p_{h}$. We have

$$
\mathcal{J}_{h}\left(p_{h}\right)=\mathcal{J}_{A}\left(p_{h}\right)+\mathcal{J}_{D}\left(p_{h}\right)+\mathcal{J}_{B}\left(p_{h}\right)
$$

where each term in the right-hand side is the Jacobian matrix of a corresponding term in (7). The term $\mathcal{J}_{A}\left(p_{h}\right)$ is easy to compute; it is a diagonal matrix with values $\left.\frac{\phi_{E} \rho}{\Delta t} \frac{\partial s}{\partial p}\right|_{p=p_{E}^{s}}$ on the diagonal. The other terms depend strongly on the discretization method. In the case of advanced discretization methods with built-in optimization or second-order upwind methods for relative permeability, the calculation of these terms becomes a non-trivial task.

\subsection{Inexact Newton method as the Picard method}

Following the framework of the inexact Newton method, we replace $\mathcal{J}_{h}$ by its approximation $\widehat{\mathcal{J}}_{h}$ and consider the following Newton-type equation for the solution increment:

$$
\widehat{\mathcal{J}}_{h}\left(p_{h}^{s}\right) \Delta p_{h}^{s+1}=-\mathcal{F}_{h}\left(p_{h}^{s}\right), \quad \Delta p_{h}^{s+1}=p_{h}^{s+1}-p_{h}^{s},
$$

where $s$ is the nonlinear iteration index. Selection of matrix $\widehat{\mathcal{J}}_{h}$ is critical for the efficiency and robustness of the nonlinear solver. Different choices for $\widehat{\mathcal{J}}_{h}$ and related linear solvers are discussed below. Note that (8) is the Picard (fixed-point) method for the modified functional $\mathcal{G}\left(p_{h}^{s}\right)$ :

$$
p_{h}^{s+1}=p_{h}^{s}-\mathcal{G}_{h}\left(p_{h}^{s}\right), \quad \mathcal{G}_{h}\left(p_{h}^{s}\right)=\widehat{\mathcal{J}}_{h}^{-1}\left(p_{h}^{s}\right) \mathcal{F}_{s}\left(p_{h}^{s}\right) .
$$

Convergence of the fixed-point method is guaranteed if $\mathcal{G}_{h}$ is the contraction operator. Although this property is difficult to check or prove in realistic models, the fixed-point method is quite popular in a wide range of applications due to simplicity of its implementation and availability of a few acceleration strategies [27, 6]. For Richards' equation we also have a simple but reliable choice for $\widehat{\mathcal{J}}_{h}$ which is defined as follows:

$$
\widehat{\mathcal{J}}_{h}^{(d)}\left(p_{h}^{s}\right)=\mathcal{J}_{A}\left(p_{h}^{s}\right)+\mathcal{D}_{h}\left(p_{h}^{s}\right) .
$$

A nonlinearity introduced by function $s(p)$ is taken care by term $\mathcal{J}_{A}\left(p_{h}^{s}\right)$. This approach is similar to the iterative procedure presented in [10] and can be considered as one of the standard technique for treating accumulation terms in Picards' method for Richards' equation. Nevertheless the nonlinear dependence of the relative permeability on the pressure is ignored.

We use superscript $(d)$ for this matrix to emphasize that this preconditioner is based on a "discretization" of the original functional. The linear operator $\widehat{\mathcal{J}}_{h}^{(d)}$ is conditionally positive definite for the presented discretization method. Note that recent progress in the mimetic technology reveals the possibility of building discretization methods that use upwinded values of the relative permeability and preserves symmetry and positive definiteness [24]. Hence, linear problems with matrix $\widehat{\mathcal{J}}_{h}^{(d)}$ can be solved efficiently with the existing multigrid methods. However, a major drawback of such a preconditioner is the slow nonlinear convergence of the Picard method in the case of strongly nonlinear relative permeability function. At best the fixed-point theory predicts a linear convergence rate when the initial guess is close to the converged solution. 


\subsection{Accelerated Picard methods}

To overcome the slow convergence of the Picard method several acceleration approaches have been proposed in the literature, including the Anderson Acceleration (AA) [2,38] and Nonlinear Krylov Acceleration (NKA) [8, 6] methods. Both the AA and NKA methods use the same design principles to extend a linear iterative scheme to the nonlinear case. This shared methodology led the authors of the NKA approach to rediscover, albeit in a different form, the iterative method presented much earlier by Anderson [2]. Specifically, the NKA approach presented in [8] is formulated in terms of differences of successive iterates and residuals. This formulation allows the basis vectors to be reused for successive iterations, and requires only one evaluation of the residual at each iteration. This same strategy was employed to improve Anderson's original formulation of the AA method in [15]. Since the cornerstone of these algorithms is the solution of the same minimization problem, in exact arithmetic they are equivalent. Nevertheless, these methods typically differ in their implementation details and apply different solution techniques to the minimization problem; a natural consequence of differing perpectives on the efficiency and robustness of the overall algorithm. Thus, both approaches lead to very similar numerical schemes that show similar performance in the published literature, as well as in our own experiments.

In this study we consider the NKA formulation as an example of an accelerated approach. This method computes the solution increment $\Delta p_{h}^{s+1}$ using at most $M \geq 1$ recent iterates to improve approximation of the Jacobian over successive iterations, $M$ is the user selected parameter. Let $m=\min (s, M)$; then, the NKA method can be written formally as follows:

$$
\Delta p_{h}^{s+1}=\mathbf{N K A}\left[\mathcal{G}_{h}\left(p_{h}^{s}\right), \mathcal{G}_{h}\left(p_{h}^{s-1}\right), \ldots, \mathcal{G}_{h}\left(p_{h}^{s-m+1}\right)\right] .
$$

When there is only one recent iterate, as at the first iteration, then the method reduces to the fixed-point iteration step, i.e. $\Delta p_{h}^{1}=-\mathcal{G}\left(p_{h}^{0}\right)$.

Let us define the following vectors:

$$
v^{s}=p_{h}^{s-1}-p_{h}^{s}, \quad w^{s}=\mathcal{G}\left(p_{h}^{s-1}\right)-\mathcal{G}\left(p_{h}^{s}\right),
$$

and the following matrices:

$$
V_{s}=\left(v_{s-m}, \ldots v_{s-1}\right), \quad W_{s}=\left(w_{s-m}, \ldots w_{s-1}\right)
$$

If $\mathcal{P}_{W}$ is the orthogonal projection onto the span of vectors $w^{s}$, then $\mathcal{G}\left(p_{h}^{s}\right)$ can be decomposed as follows:

$$
\mathcal{G}\left(p_{h}^{s}\right)=\mathcal{P}_{W} \mathcal{G}\left(p_{h}^{s}\right)+\left(I-\mathcal{P}_{W}\right) \mathcal{G}\left(p_{h}^{s}\right) .
$$

Note that $\left(I-\mathcal{P}_{W}\right) \mathcal{G}\left(p_{h}^{s}\right)$ is orthogonal to all vectors $w^{s}$. The key idea of the NKA method is to approximate the action of the Jacobian matrix on the projection term and apply the fixed-point update for the orthogonal term. To compute the above decomposition, we solve the following least squares problem:

$$
\min _{\gamma=\left(\gamma_{0}, \ldots, \gamma_{s-1}\right)^{T}}\left\|\mathcal{G}\left(p_{h}^{s}\right)-W_{s} \gamma\right\|_{2}
$$

to obtain

$$
\Delta p_{h}^{s+1}=V_{s} \gamma+\mathcal{G}\left(p_{h}^{s}\right)-W_{s} \gamma
$$


The performance of the NKA method hinges on the properties of matrix $\widehat{\mathcal{J}}_{h}\left(p_{h}^{s}\right)$. In practice, to increase computational efficiency, this matrix is recomputed every few nonlinear iterations based on an empirical algorithm which complicates the convergence analysis. If $\widehat{\mathcal{J}}_{h}\left(p_{h}^{s}\right)$ is a poor approximation of the Jacobian matrix (in some metric), then the NKA method will require more iterations to convergence.

Remark 3.1. The efficacy of the AA method for variably saturated flows was studied in [27]. Since $A A$ and NKA methods are similar in many aspects we do not present the description and numerical results for the AA method. We also observe that the benefits of a new preconditioning strategy extends to the AA method as well.

\subsection{New preconditioner and novel nonlinear method}

To find more accurate and stable approximation of the Jacobian matrix we consider a novel methodology that is the reverse of the conventional approach. We start with the derivation of the continuum Jacobian operator for functional $\mathcal{F}$ using the Fréchet derivative [13]. The mathematical definition of the Fréchet derivative $\mathcal{J}\left(p^{s}\right)$ of $\mathcal{F}$ at point $p^{s}$ (superscript $s$ is used only to emphasize connection with the discrete Jacobian) acting on a small increment function $\delta p$ states that

$$
\left\|\mathcal{F}\left(p^{s}+\delta p\right)-\mathcal{F}\left(p^{s}\right)-\mathcal{J}\left(p^{s}\right) \delta p\right\|_{Y}=o\left(\|\delta p\|_{X}\right)
$$

in appropriate Banach spaces $X$ and $Y$. To avoid technical details, we assume for a moment that all functions are sufficiently smooth and $\phi$ and $\rho$ are constants. Then, $X$ and $Y$ are the spaces of continuously differentiable (in space and time) functions. Let

$$
\mathcal{J}\left(p^{s}\right) \delta p=\phi \rho \frac{\partial s}{\partial p}\left(p^{s}\right) \frac{\partial \delta p}{\partial t}-\operatorname{div}\left(\mathbf{K} k\left(p^{s}\right) \frac{\rho}{\mu} \nabla \delta p\right)-\operatorname{div}\left(\mathbf{K} \frac{\partial k}{\partial p}\left(p^{s}\right) \frac{\rho}{\mu}\left(\nabla p^{s}-\rho \mathbf{g}\right) \delta p\right) .
$$

Using the Taylor expantion of saturation and relative permeability functions around point $p^{s}$ we can easily verify that

$$
\mathcal{F}\left(p^{s}+\delta p\right)-\mathcal{F}\left(p^{s}\right)-\mathcal{J}\left(p^{s}\right) \delta p=\Psi\left((\delta p)^{2}\right),
$$

where $\Psi$ is the bounded operator from $X$ to $Y$. The result can be generalized to problems with less regularity using appropriate Sobolev spaces $X$ and $Y$; however, the analysis is more involved.

The second term in the right-hand side of (9) is the elliptic operator with respect to $\delta p$ and is similar to the diffusion term in the original nonlinear PDE but with the lagged relative permeability. The last term is the advection operator with respect to $\delta p$. Indeed, we can rewrite it as

$$
\operatorname{div}\left(\mathbf{V}^{s} \delta p\right), \quad \mathbf{V}^{s}=-\mathbf{K} \frac{\partial k}{\partial p}\left(p^{s}\right) \frac{\rho}{\mu}\left(\nabla p^{s}-\rho \mathbf{g}\right)=\mathbf{q}^{s} \frac{\partial k}{\partial p}\left(p^{s}\right) \frac{\rho}{k\left(p^{s-1}\right)} .
$$

Note that velocity $\mathbf{V}^{s}$ is the Darcy velocity scaled by the non-negative factor due to (2). This term may dominate the diffusion part many times for low capillary pressures. Thus, its inclusion in a preconditioner may influence enormously the convergence of the nonlinear 
solver. It is important to note that to achieve a positive impact on the convergence rate, we need a stable discretization of this term.

Equation (9) guides our selection of numerical methods for its discretization by allowing only those that have proper mathematical properties. For the first term, we use the backward Euler scheme. For the the second term, we use the MFD scheme. For the last term, the advection operator, we use the cell-centered upwind finite volume scheme. Then, the discrete form of this operator becomes

$$
\widehat{\mathcal{J}}_{h}^{(l)}\left(p^{s}\right) \delta p_{h}=\mathcal{J}_{A}\left(p_{h}^{s}\right) \delta p_{h}+\mathcal{D}_{h}\left(p_{h}^{s}\right) \delta p_{h}+\operatorname{div}^{h, 2}\left(\mathbf{V}_{h}^{s} \delta p_{h}\right)
$$

where $\mathbf{V}_{h}^{s}$ is the following special approximation of velocity $\mathbf{V}^{s}$ :

$$
\mathbf{V}_{h}^{s}=-\mathbf{q}_{h}^{s} \frac{\partial k}{\partial p}\left(p_{h}^{s}\right) \frac{\rho}{k\left(p_{h}^{s}\right)} .
$$

and $\frac{\partial k}{\partial p}\left(p_{h}^{s}\right), k\left(p_{h}^{s}\right)$ on mesh edges are computed using the upwind approach. Note that we changed the iteration level of the relative permeability which gives the same error as the linearization procedure. The superscript $l$ is used to emphasize that the preconditioner $\widehat{\mathcal{J}}_{h}^{(l)}$ is derived based on the linearization of the original functional.

Remark 3.2. Note that the semi-discrete form of operator in (9) is positive definite under the point-wise constraint

$$
\frac{\rho \phi}{\Delta t} \frac{\partial s}{\partial p}\left(p^{s}\right)+\frac{1}{2} \operatorname{div} \mathbf{V}^{s} \geq 0
$$

If $\mathcal{D}_{h}\left(p_{h}^{s}\right)$ is positive definite, the upwind scheme guarantees that matrix $\widehat{\mathcal{J}}_{h}^{(l)}\left(p^{s}\right)$ is the positive definiteness under the discrete from of this constraint.

Using $\widehat{\mathcal{J}}^{(l)}\left(p^{s}\right)$ in the Picard method leads to a novel nonlinear solver. One step of this solver can be written as the solution of the singularly perturbed diffusion-advection equation:

$$
\phi \rho \frac{\partial s}{\partial p}\left(p_{h}^{s}\right) \frac{p_{h}^{s+1}-p_{h}^{s}}{\Delta t}+\phi \rho \frac{s\left(p_{h}^{s}\right)-s\left(p_{h}^{0}\right)}{\Delta t}-\operatorname{div}^{h, 1}\left(\rho \mathbf{q}_{h}^{s+1}\right)+\operatorname{div}^{h, 2}\left(\mathbf{V}_{h}^{s}\left(p_{h}^{s+1}-p_{h}^{s}\right)\right)=0
$$

This solver has a few interesting properties. Proper selection of the discrete operators allows us to control properties of the next iterate $p_{h}^{s+1}$. For instance, nonlinear finite volume schemes for the diffusion operator can be used to guarantee solution monotonicity and/or positivity. The last term can be controlled with additional limiters to reduce the CFL-type condition for the operator ellipticity. Observe that this term vanishes as $p_{h}^{s}$ converges to the solution. Although formula (12) is very beneficial for theoretical analysis and understanding of the new solver, in our numerical experiments it is implemented as the Picard method:

$$
p_{h}^{s+1}=p_{h}^{s}-\left(\widehat{\mathcal{J}}_{h}^{(l)}\left(p_{h}^{s}\right)\right)^{-1} \mathcal{F}\left(p_{h}^{s}\right) .
$$




\subsection{Jacobian free Newton Krylov (JFNK)}

Among nonlinear solvers which avoid generating the Jacobian matrix the Jacobian-Free Newton Krylov (JFNK) method is probably the most popular one. The combination of the classical Newton method and a Krylov-type iterative method for solving linear systems is the basis of the JFNK method. Krylov iterative methods for solving large linear systems were proposed in 1950's [18]. These are the projection methods for solving $A x=b$ using Krylov spaces $K_{j}$ :

$$
K_{j}=\operatorname{span}\left(r, A r, A^{2} r, \ldots A^{j-1} r\right),
$$

where $r=b-A x$ is the residual vector. The key property of these methods is that no explicit knowledge of matrix entries is required. Only the matrix-vector operation has to be defined to carry out an iteration process.

We apply the Krylov-type generalized minimal residual (GMRES) method to find an approximate solution of the linear system

$$
\mathcal{J}_{h}\left(p_{h}^{s}\right) \Delta p_{h}^{s+1}=-\mathcal{F}_{h}\left(p_{h}^{s}\right)
$$

where $\mathcal{J}_{h}\left(p_{h}^{s}\right)$ is the analytic Jacobian matrix that we do not want to compute. To define the action of the Jacobian on a vector $v_{h}$, we employ the finite difference approximation:

$$
\mathcal{J}_{h}\left(p_{h}^{s}\right) v_{h}=\frac{\mathcal{F}_{h}\left(p_{h}^{s}+\varepsilon v_{h}\right)-\mathcal{F}_{h}\left(p_{h}^{s}\right)}{\varepsilon} .
$$

Obviously, accuracy of this approximation depends on the choice of $\varepsilon$ and translates into stability of the GMRES method. There exists several strategies for selecting $\varepsilon$ that are described in [22]. In this paper we use the approach presented originally in [32]:

$$
\varepsilon=\frac{\sqrt{1+\left\|p_{h}^{s}\right\|_{2}}}{\left\|v_{h}\right\|_{2}} \sqrt{\varepsilon_{\text {machine }}}
$$

where $\varepsilon_{\text {machine }}$ is the computer round-off tolerance.

Since the matrix-vector product is computed approximately, there is no need to solve equation (13) with high accuracy. In practice, on each time step of the inexact Newton solver, the following convergence criterion is used:

$$
\left\|\mathcal{J}_{h}\left(p_{h}^{s}\right) \Delta p_{h}^{s+1}+\mathcal{F}_{h}\left(p_{h}^{s}\right)\right\|_{2}<\varepsilon_{l i n}\left\|\mathcal{F}_{h}\left(p_{h}^{s}\right)\right\|_{2}
$$

Convergence of inexact Newton solvers is analyzed in [12].

To accelerate convergence of the JFNK, a spectrally equivalent preconditioner is needed. The JFNK framework allows one to rely on existing codes and "physics-based " concepts such as operator splitting to build simple and physics motivated preconditioners. This approach results in a discretization of the original PDE with lagged coefficients. In many cases this approximation of the Jacobian matrix is not accurate enough. For Richards' equation the standard approach means that the preconditioner is build using matrix $\widehat{\mathcal{J}}_{h}^{(d)}\left(p_{h}^{s}\right)$. In the next section we compare this choice with $\widehat{\mathcal{J}}_{h}^{(l)}\left(p_{h}^{s}\right)$. 


\section{Numerical experiments}

In the numerical experiments we study performance of the nonlinear solvers in two different simulation regimes: calculation of a steady-state solution and a transient flow driven by time-dependent boundary conditions. The performance of any nonlinear solver depends highly on its control parameters. We strive to make a fair comparison of the performance of preconditioners and nonlinear solvers by optimizing there parameters to minimize overall CPU time. All numerical experiments were performed using the production code Amanzi, which is the multi-process of the Advanced Simulation Capability for Environmental Management (ASCEM) program [1].

The stopping criterion is one of the important control parameters. Although in many applications this criterion is based on the $L_{2}$ norm of the nonlinear residual, for Richards' equation this criterion is typically too weak. The importance of the fine-scale solution dynamics requires a stronger criterion that is the combination of the infinity norms of the normalized nonlinear residual and of the solution increment:

$$
\left\|e_{h}\right\|_{\infty}:=\max \left(\left\|\frac{\left|\Delta p_{h}\right|}{\left|p_{h}-p_{a t m}\right|+p_{a t m}}\right\|_{\infty},\left\|\frac{\Delta t}{\phi \rho} \mathcal{F}_{h}\right\|_{\infty}\right) \leq \varepsilon_{n o n} .
$$

This nonlinear tolerance is set to $\varepsilon_{n o n}=10^{-6}$ in all tests which implies that the pressure error is about $0.1[\mathrm{~Pa}]$ and the saturation error is about $10^{-6}$. This convergence criterion is essential for obtaining a reliable numerical solution and also implemented in the generalpurpose simulator for subsurface flow and transport STOMP [39]. Unfortunately, the convergence theory in the maximum norm is limited for both discretization methods and nonlinear solvers, so the numerical results are the only available tool for comparing different nonlinear solvers.

To increase the convergence region of nonlinear solvers, we incorporate a local damping procedure into their algorithms. A similar approach has been implemented in the subsurface flow and reactive transport code PFLOTRAN [31]. First, this relaxation procedure modifies solution increment $\Delta p_{h}^{s+1}$ such that the corresponding saturation change in every cell is bounded by $\Delta s_{\max }=0.25$. Second, it performs a local relaxation in cells which move from a partially saturated state into the fully saturated state. For these cells, we set $\Delta p_{E}^{s+1}:=\alpha \Delta p_{E}^{s+1}$ where $\alpha=0.5$. Based on our experience these techniques improve robustness of the nonlinear solvers by increasing the number of successful time steps although for some time steps the number of nonlinear iterations may slightly grow.

To improve overall computational performance, the tolerance of linear solvers is defined relative to the tolerance of nonlinear solvers. Moreover, the choice of the left or right preconditioning strategy also may affect performance of a linear solver. In the numerical tests presented below the left preconditioner approach is implemented. More precisely, we set $\left\|M^{-1} r_{\text {lin }}\right\|_{2} \leq 10^{-2}\left\|M^{-1} \mathcal{F}_{h}\right\|_{2}$, where $\mathcal{F}_{h}$ is the current nonlinear residual, $r_{\text {lin }}$ is the residual of a linear solver and $M^{-1}$ is the preconditioner defined by the action of five V-cycles of the algebraic multigrid solver BoomerAMG (Hypre library, [16]) applied to $\widehat{\mathcal{J}}_{h}^{(d)}$ or $\widehat{\mathcal{J}}_{h}^{(l)}$ matrices. We also tested the right preconditioning approach in some numerical experiments and observed that the left preconditioner approach lead to more linear iterations, but fewer nonlinear iterations and time steps than the right preconditioner strategy. Overall CPU time difference was within $10 \%$ range, but it was inconclusive what strategy is the fastest. 
In the numerical experiments described below we study the impact of the new preconditioner $\widehat{\mathcal{J}}_{h}^{(l)}$ on the JFNK, NKA, and Picard solvers. All tests were performed on one core of a workstation with $2.1 \mathrm{GHz}$ AMD Opteron(TM) Processor 6272. In the first experiment, Richards equation is discretized on a uniform orthogonal grid using the MFD method. In the second and third experiments, the computational domain has a tilted top surface and sloping layers of variable thickness to represent the aquifer stratigraphy of more realistic subsurface structures. In all cases the mesh is fitted to three geological layers with different porosities, permeabilities and water retention models.

\subsection{Rectangular domain and orthogonal mesh}

In the first experiment, we consider the rectangular domain $\Omega=[0,200 \mathrm{~m}] \times[0,100 \mathrm{~m}]$. Richards' equation is discretized on two uniform rectangular meshes with $64 \times 64$ and $128 \times 128$ mesh cells, respectively. The geometric model has three layers with different material properties which are summarized in Table 1.

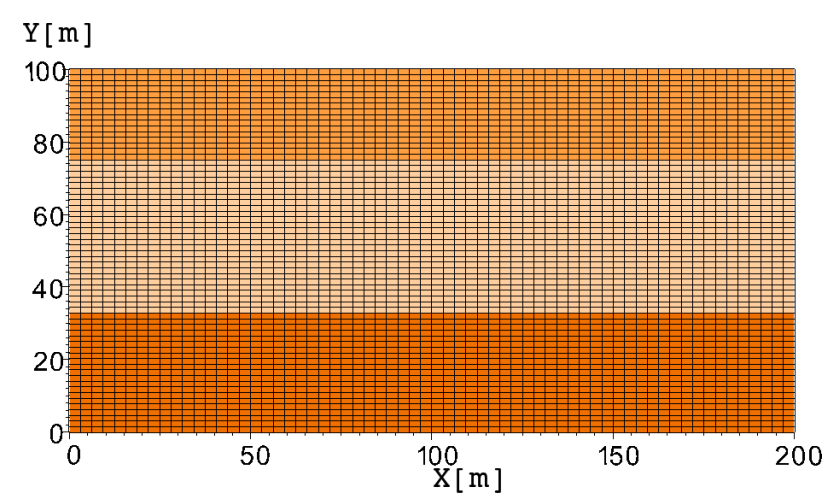

Figure 1: The first experiment: the rectangular mesh matching interfaces between three different soils.

\begin{tabular}{r|c|c|c|c|c} 
& $\mathbf{K}\left[\mathrm{m}^{2}\right]$ & $\phi[-]$ & $\alpha\left[\mathrm{Pa}^{-1}\right]$ & $m[-]$ & $n[-]$ \\
\hline Top layer & $2.07 \cdot 10^{-11}$ & 0.2340 & $2.0674 \cdot 10^{-4}$ & 0.3006 & 1.4298 \\
Middle layer & $1.99 \cdot 10^{-12}$ & 0.4082 & $1.9467 \cdot 10^{-4}$ & 0.2294 & 1.2977 \\
Bottom layer & $6.93 \cdot 10^{-11}$ & 0.2206 & $2.0260 \cdot 10^{-3}$ & 0.2136 & 1.2716
\end{tabular}

Table 1: Isotropic absolute permeability $(\mathbf{K})$, porosity $(\phi)$ and van Genuchten parameters $\alpha, m$ and $n$ for the water retention models in different layers.

\subsubsection{Steady-state calculation}

The steady-state solution can be computed either by evolving the system over a long period of time or through the direct solution of the nonlinear system. We employ the former strategy: the system is evolved starting with some initial pressure profile at time 0 to the final state at year 1956 which we call the steady-state solution. To make this approach computationally feasible a variable time stepping strategy is essential. Here, and in the subsequent experiments we apply a heuristic time stepping strategy based on the 
number of nonlinear iterations, $n_{n l}$, on each time step. The next time step is derived by the following formula:

$$
\Delta t_{n+1}:=\xi \Delta t_{n} \quad \text { where } \quad \xi=\left\{\begin{array}{lll}
1.25 & \text { if } n_{n l} \leq n_{\min } \\
1 & \text { if } \quad n_{\min }<n_{n l} \leq n_{\max } \\
0.8 & \text { if } \quad n_{\max }<n_{n l}
\end{array}\right.
$$

and parameters $n_{\min }$ and $n_{\max }$ are set as follows: $n_{\min }=10, n_{\max }=15$. This strategy is often used when error estimates are not available or are too expensive to calculate. The convergence rate of the Picard or NKA solver depends on the contraction properties of fixed point operators $\mathcal{G}$ and hence on the used time step. The heuristic time step strategy drives a simulation to use a time step that reflects contraction properties of $\mathcal{G}$. The selected values, $n_{\min }=10$ and $n_{\max }=15$, were found experimentally to minimize the total number of nonlinear iterations in a simulation.

We prescribe the constant mass influx on the top boundary of $\Omega$ which is equal to $1.1071 \cdot 10^{-7}\left[\mathrm{~kg} \cdot \mathrm{m} \cdot \mathrm{s}^{-1}\right]$ and the constant atmospheric pressure $p_{\text {atm }}$ on the bottom boundary. On the remaining boundary we use the no-flow boundary condition. The initial guess is set to be the clipped hydrostatic pressure:

$$
p(x, y)=\max \left\{p_{a t m}-\rho g y, 0.5 p_{a t m}\right\}
$$

where $g$ is the gravity magnitude, $\rho$ is the liquid density, $y$ is the height above the bottom boundary of the computational domain. This clipping of the hydrostatic pressure improves convergence of the nonlinear solvers since it eliminates large solution gradients when the wetting front propagates inside the domain.

In Table 2 we present the total number of successful and failed time steps and the total number of nonlinear iterations (in successful and failed time steps) performed by each solver. The time step is referred to as failed if the nonlinear solver is unable to converge within $n_{\max }$ nonlinear iterations. It is important to note that good performance of a nonlinear solver may lead to failed time steps. It happens when a time step increases faster than a nonlinear solver can handle. (In these situations the parameters of variable time stepping strategy should be adjusted.) The total CPU time required to reach the steady-state solution is shown in Table 3 . We observe that the new preconditioner $\widehat{\mathcal{J}}_{h}^{(l)}$ improves the performance of all solvers significantly. Although the number of time steps and nonlinear iterations for the JFNK solver is quite low for both preconditioners, this solver is much slower than the others. We attribute this to the fact that the matrix-vector product in the GMRES is approximated with Gâteaux derivative. On each linear iteration the approximate matrix-vector product corresponds effectively to a slightly different matrix which slows down the GMRES convergence. Such linear method is formally called as the flexible GMRES (FGMRES) although algorithmically it is equivalent to the GMRES method. The FGMRES method does not modify the basic residual minimization idea of the GMRES method, but in the FGMRES framework it is not possible to prove the convergence properties similar to the GMRES method. In the Picard and NKA solvers, the matrix is given explicitly which leads to the faster convergence of the GMRES method. The NKA and Picard methods reduce the number of time steps and the number of nonlinear iterations close to the JFNK solver numbers but keep the computational cost of each nonlinear iteration lower. The overall speedup in CPU times is about 3-12 times. 


\begin{tabular}{c|c|c|c|c|c|c} 
& \multicolumn{3}{|c|}{ \# successful time steps ( \# failed time steps) } \\
\cline { 2 - 7 } & \multicolumn{2}{|c|}{ JFNK } & \multicolumn{2}{|c|}{ NKA } & \multicolumn{2}{c}{ Picard } \\
\cline { 2 - 7 } & $\widehat{\mathcal{J}}_{h}^{(d)}$ & $\widehat{\mathcal{J}}_{h}^{(l)}$ & $\widehat{\mathcal{J}}_{h}^{(d)}$ & $\widehat{\mathcal{J}}_{h}^{(l)}$ & $\widehat{\mathcal{J}}_{h}^{(d)}$ & $\widehat{\mathcal{J}}_{h}^{(l)}$ \\
\hline mesh 64x64 & $53(0)$ & $53(0)$ & $145(0)$ & $55(0)$ & $247(64)$ & $53(0)$ \\
\# nonlinear itr & 197 & 202 & 1329 & 262 & 3147 & 223 \\
\hline mesh 128x128 & $53(0)$ & $53(0)$ & $126(0)$ & $57(0)$ & $335(128)$ & $53(0)$ \\
\# nonlinear itr & 203 & 214 & 1150 & 290 & 4781 & 228
\end{tabular}

Table 2: The total number of successful and failed time steps and the nonlinear iterations required to calculate the steady-state solution.

\begin{tabular}{c|c|c|c|c|c|c}
\multirow{2}{*}{ mesh } & \multicolumn{5}{|c|}{ CPU times (sec) } \\
\cline { 2 - 7 } & \multicolumn{2}{|c|}{ JFNK } & \multicolumn{2}{|c|}{ NKA } & \multicolumn{2}{c}{ Picard } \\
\cline { 2 - 7 } & $\widehat{\mathcal{J}}_{h}^{(d)}$ & $\widehat{\mathcal{J}}_{h}^{(l)}$ & $\widehat{\mathcal{J}}_{h}^{(d)}$ & $\widehat{\mathcal{J}}_{h}^{(l)}$ & $\widehat{\mathcal{J}}_{h}^{(d)}$ & $\widehat{\mathcal{J}}_{h}^{(l)}$ \\
\hline $64 \times 64$ & 411.8 & 70.6 & 153.6 & 28.0 & 250.0 & 23.7 \\
$128 \times 128$ & 2197.8 & 363.6 & 405.9 & 146.1 & 1690.5 & 115.8
\end{tabular}

Table 3: Computational cost of the steady-state calculation.

\subsubsection{Transient flow driven by boundary conditions}

In the transient regime, we modify the boundary conditions on the top surface. We select the subdomain $\Gamma_{\text {inf }}=\{(x, y): 60 \mathrm{~m} \leq x \leq 120 \mathrm{~m}$ and $y=100 \mathrm{~m}\}$ where the infiltration rate is changing with time. This region models a seepage basin for liquid waste. At year 1956, the mass flux over $\Gamma_{\text {inf }}$ is set to the new constant rate of $0.002\left[\mathrm{~kg} \cdot \mathrm{m} \cdot \mathrm{s}^{-1}\right]$. At time $1956.08[\mathrm{y}]$ the active phase of the infiltration is turned off and the mass flux is reset to the original value $1.1071 \cdot 10^{-7}\left[\mathrm{~kg} \cdot \mathrm{m} \cdot \mathrm{s}^{-1}\right]$. The initial state for this simulation is the steady-state solution computed in the previous section.

The snapshots of the pressure at different moments in time are shown in Fig. 2. As expected, the solution dynamics is dominated by gravity; the plume is moving vertically with small horizontal diffusion. The total number of time steps and the CPU time required to reach the final state at year 1959 are presented in Tables 4-5. Since on every time step the nonlinear solvers start with a physically meaningful initial guess, the impact of the new preconditioner is less than in the steady-state calculation. Still, the Picard and NKA solvers significantly outperform the JFNK solver due to the faster convergence of the GMRES method.

\subsection{Non-orthogonal geometry and disturbed mesh}

In this section we present the results of numerical experiments on two distorted quadrilateral meshes. The geometric model is shown in Fig. 3 and has again three layers with different material properties summarized in Table 1. The logically structured mesh is mapped onto this geometric model.

For a distorted mesh, the classical finite volume scheme is no longer accurate and we have to employ an advanced discretization scheme, such as the MFD method [17]. In 

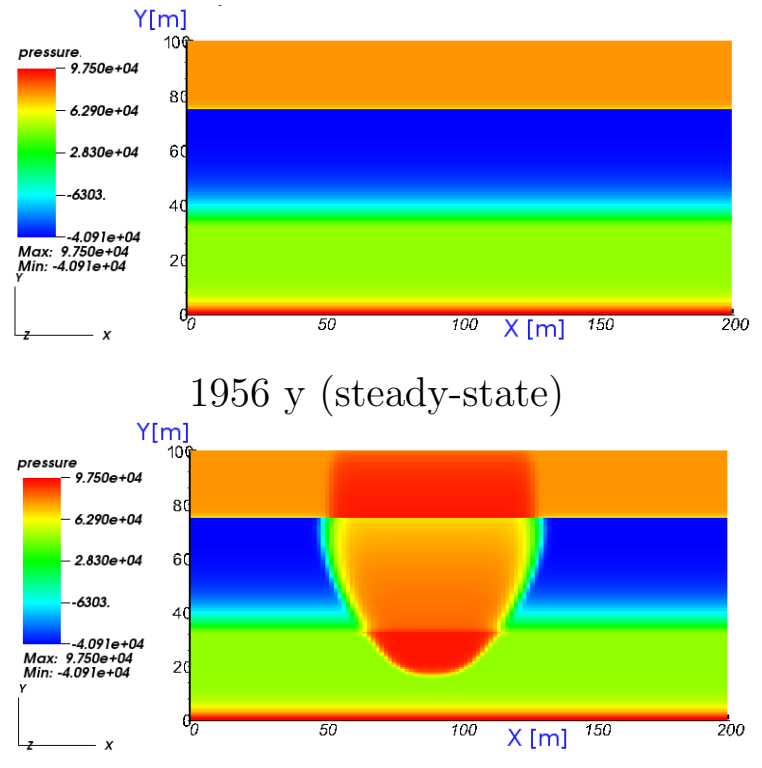

1958 year

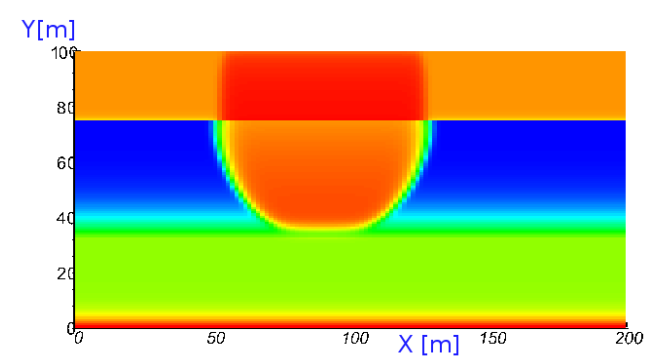

1957 year

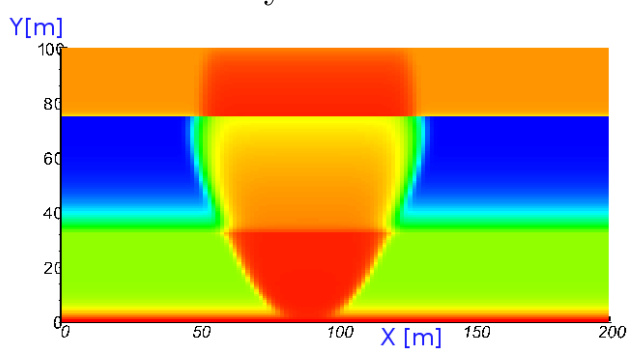

1959 year

Figure 2: Pressure profile at years 1956, 1957, 1958 and 1959.

\begin{tabular}{c|c|c|c|c|c|c} 
& \multicolumn{4}{|c}{ \# successful time steps ( \# failed time steps) } \\
\cline { 2 - 7 } & \multicolumn{2}{|c|}{ JFNK } & \multicolumn{2}{|c|}{ NKA } & \multicolumn{2}{c}{ Picard } \\
\cline { 2 - 7 } & $\widehat{\mathcal{J}}_{h}^{(d)}$ & $\widehat{\mathcal{J}}_{h}^{(l)}$ & $\widehat{\mathcal{J}}_{h}^{(d)}$ & $\widehat{\mathcal{J}}_{h}^{(l)}$ & $\widehat{\mathcal{J}}_{h}^{(d)}$ & $\widehat{\mathcal{J}}_{h}^{(l)}$ \\
\hline mesh 64x64 & $104(7)$ & $104(7)$ & $392(0)$ & $292(4)$ & $572(8)$ & $223(5)$ \\
\# nonlinear itr & 668 & 668 & 3117 & 2138 & 5435 & 1188 \\
\hline mesh 128x128 & $137(28)$ & $138(29)$ & $684(3)$ & $389(11)$ & $941(1)$ & $256(8)$ \\
\# nonlinear itr & 1138 & 1169 & 6528 & 3428 & 9800 & 1685
\end{tabular}

Table 4: Transient flow on the rectangular meshes: the number of successful and failed time steps and the total number of nonlinear iterations.

\begin{tabular}{c|c|c|c|c|c|c}
\multirow{2}{*}{ mesh } & \multicolumn{5}{|c|}{ CPU times (sec) } \\
\cline { 2 - 7 } & \multicolumn{2}{|c|}{ JFNK } & \multicolumn{2}{|c}{ NKA } & \multicolumn{2}{c}{ Picard } \\
\cline { 2 - 7 } & $\widehat{\mathcal{J}}_{h}^{(d)}$ & $\widehat{\mathcal{J}}_{h}^{(l)}$ & $\widehat{\mathcal{J}}_{h}^{(d)}$ & $\widehat{\mathcal{J}}_{h}^{(l)}$ & $\widehat{\mathcal{J}}_{h}^{(d)}$ & $\widehat{\mathcal{J}}_{h}^{(l)}$ \\
\hline $64 \times 64$ & 841.5 & 303.7 & 211.6 & 192.7 & 357.0 & 111.2 \\
$128 \times 128$ & 10956.8 & 3805.6 & 2122.9 & 1363.1 & 3500.6 & 813.6
\end{tabular}

Table 5: Computational cost of the transient flow on the rectangular meshes.

comparison with the finite volume scheme, the mimetic scheme has two types of pressure unknowns which makes convergence of nonlinear solvers more challenging. Since we demonstrated previously with for the orthogonal geometry, the NKA and Picard solvers significantly outperform the JFNK solver. Therefore, in this section we are not performing numerical experiments with the JFNK solver.

Similarly to the previous section, first, we compute a steady-state solution through 


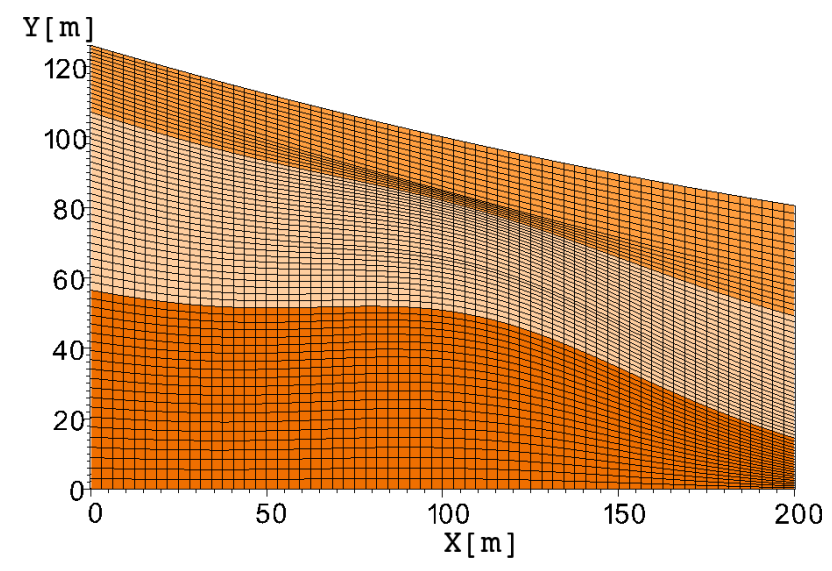

Figure 3: The layered structure of the computational domain in the second experiment.

evolving the model from 0 year till 1956 year. The vertical profiles of the steady-state solution at $x=100 \mathrm{~m}$ are shown in Fig. 4. Note that pressure is monotone in each soil which satisfies the maximum principle for Richards' equation.
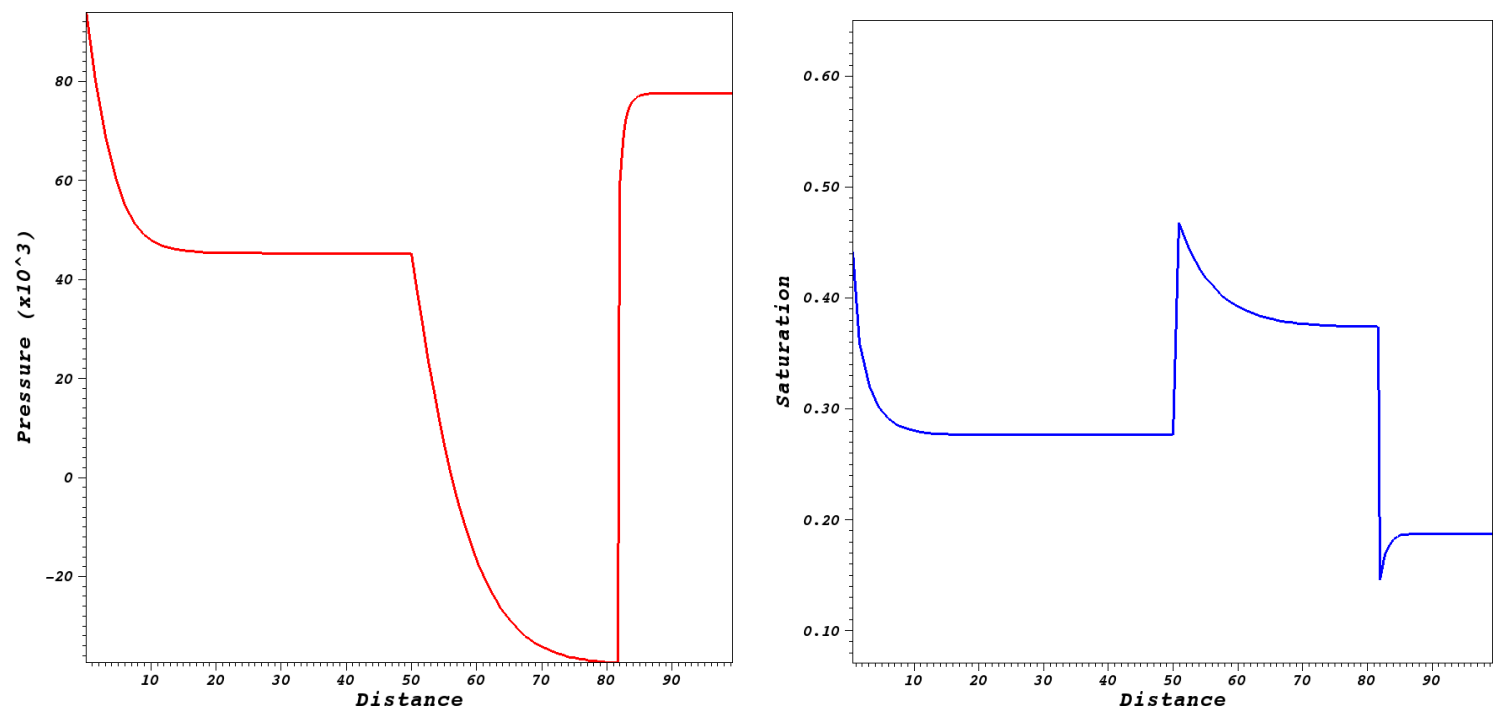

Figure 4: The vertical profile of pressure(left) and saturation(right) in the steady-state solution at $x=$ $100 \mathrm{~m}$.

In Tables 6-7 the number of successful and failed time steps, the total number of nonlinear iterations, and CPU times are presented. The numerical results demonstrate that the linearized preconditioner $\widehat{\mathcal{J}}_{h}^{(l)}$ improves the performance of both nonlinear solvers significantly. As it was observed in the previous section, convergence of the Picard method with the standard preconditioner $\widehat{\mathcal{J}}_{h}^{(d)}$ could be an issue. Acceleration methods, such as the NKA solver, alleviate this issue and improve nonlinear convergence 6-7 times based on the total number of nonlinear iterations. It would be natural to expect the moderate impact of the new preconditioner on such efficient solvers. However, the new preconditioner reduced the computational cost 5 times for the NKA solver and about 40 times for the Picard solver. 


\begin{tabular}{c|c|c|c|c} 
& \multicolumn{3}{|c}{ \# successful time steps( \# failed time steps) } \\
\cline { 2 - 5 } & \multicolumn{2}{|c|}{ NKA } & \multicolumn{2}{c}{ Picard } \\
\cline { 2 - 5 } & $\widehat{\mathcal{J}}_{h}^{(d)}$ & $\widehat{\mathcal{J}}_{h}^{(l)}$ & $\widehat{\mathcal{J}}_{h}^{(d)}$ & $\widehat{\mathcal{J}}_{h}^{(l)}$ \\
\hline mesh 64x64 & $132(0)$ & $57(0)$ & $529(155)$ & $53(0)$ \\
\# nonlinear itr & 1209 & 297 & 8318 & 234 \\
\hline mesh 128x128 & $223(0)$ & $61(0)$ & $646(376)$ & $55(2)$ \\
\# nonlinear itr & 2171 & 340 & 12811 & 269
\end{tabular}

Table 6: Steady-state calculation on the non-orthogonal geometry: the number of successful and failed time steps and the total number of nonlinear iterations.

\begin{tabular}{c|c|c|c|c}
\multirow{2}{*}{ mesh } & \multicolumn{3}{|c}{ CPU times (sec) } \\
\cline { 2 - 5 } & \multicolumn{2}{|c|}{ NKA } & \multicolumn{2}{c}{ Picard } \\
\cline { 2 - 5 } & $\widehat{\mathcal{J}}_{h}^{(d)}$ & $\widehat{\mathcal{J}}_{h}^{(l)}$ & $\widehat{\mathcal{J}}_{h}^{(d)}$ & $\widehat{\mathcal{J}}_{h}^{(l)}$ \\
\hline $64 \times 64$ & 143.95 & 43.46 & 1044.12 & 36.23 \\
$128 \times 128$ & 1226.70 & 230.38 & 7259.44 & 189.01
\end{tabular}

Table 7: Computational cost of the steady-state calculation on the non-orthogonal geometry.

Let us now consider the transient regime. Similarly to the orthogonal test case, we define a special infiltration area on the top surface and set up dynamic infiltration boundary conditions on it. The transient simulation runs for 3 years.

In Fig. 5, we show the snapshots of the pressure at different time moments. In contrast to the orthogonal geometry, there exist a slight horizontal flow that shifts the plume center to the right. In these experiments, the new preconditioner $\widehat{\mathcal{J}}_{h}^{(l)}$ again shows significant performance improvement over the standard preconditioner $\widehat{\mathcal{J}}_{h}^{(d)}$. The total number of time steps and the CPU times are presented in Tables 8-9. The transient regime is very challenging for the Picard solver with $\widehat{\mathcal{J}}_{h}^{(d)}$; the very large number of nonlinear iterations is caused by the large percentage of failed time steps. However, with the new preconditioner, the Picard solver slightly outperforms the NKA solver which means that the acceleration technique does not provide much benefit here.

\begin{tabular}{c|c|c|c|c}
\multirow{2}{*}{} & \multicolumn{2}{|c}{ \# successful time steps ( \# failed time steps) } \\
\cline { 2 - 5 } & $\widehat{\mathcal{J}}_{h}^{(d)}$ & $\widehat{\mathcal{J}}_{h}^{(l)}$ & $\widehat{\mathcal{J}}_{h}^{(d)}$ & $\widehat{\mathcal{J}}_{h}^{(l)}$ \\
\hline mesh 64x64 & $616(39)$ & $363(37)$ & $2051(1343)$ & $323(39)$ \\
\# nonlinear itr & 5801 & 3046 & 21044 & 2423 \\
\hline mesh 128x128 & $1030(90)$ & $530(75)$ & $3484(1732)$ & $430(61)$ \\
\# nonlinear itr & 10528 & 5434 & 41583 & 3919
\end{tabular}

Table 8: Transient flow on the non-orthogonal geometry: the number of successful and failed time steps and the total number of nonlinear iterations. 


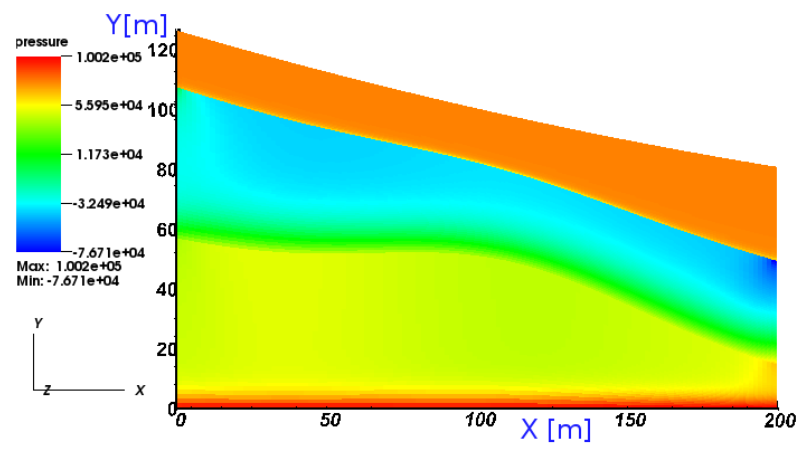

1956 year (steady-state)

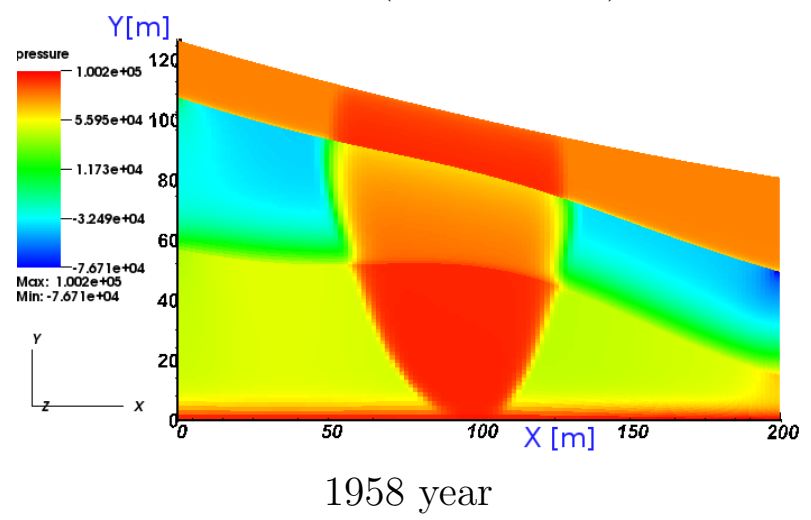

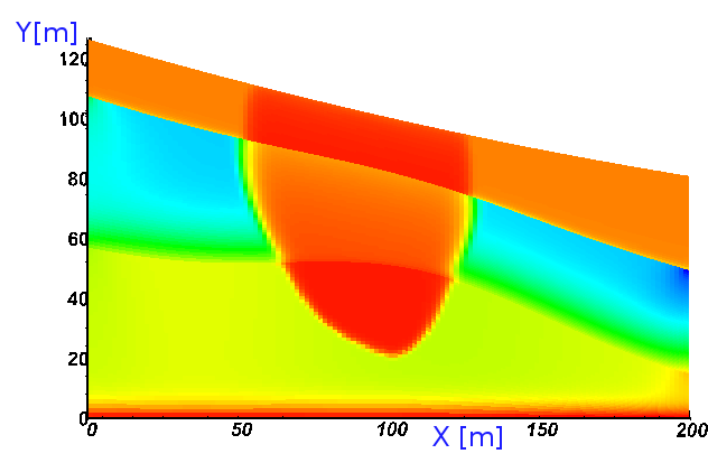

1957 year

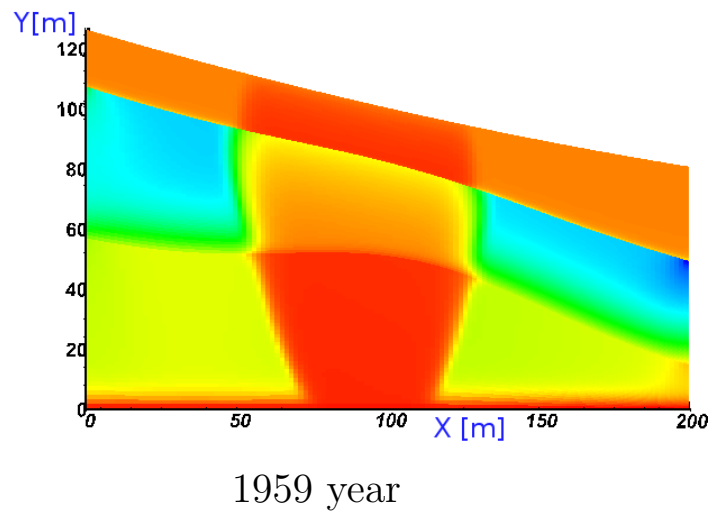

Figure 5: Pressure profile at years 1956, 1957, 1958 and 1959.

\begin{tabular}{c|c|c|c|c}
\multirow{2}{*}{ mesh } & \multicolumn{3}{|c}{ CPU times (sec) } \\
\cline { 2 - 5 } & \multicolumn{2}{|c|}{ NKA } & \multicolumn{2}{|c}{ Picard } \\
\cline { 2 - 5 } & $\widehat{\mathcal{J}}_{h}^{(d)}$ & $\widehat{\mathcal{J}}_{h}^{(l)}$ & $\widehat{\mathcal{J}}_{h}^{(d)}$ & $\widehat{\mathcal{J}}_{h}^{(l)}$ \\
\hline 64x64 & 559.7 & 334.5 & 1910.0 & 299.9 \\
$128 \times 128$ & 4997.1 & 2661.7 & 15368.6 & 2034.3
\end{tabular}

Table 9: Computational cost of the transient flow on the non-orthogonal geometry.

\subsection{Sensitivity to the parameters of the water retention models}

The nonlinearity of the solution is strongly influenced by the parameters of van Genuchten and Mualem models (3). Many researches point out that nonlinear solvers may behave differently when $n>2$ or $n<2$. The main reason for this behavior is a discontinuity of the derivative of $k_{r}$ at $p=p_{a t m}$ if $n<2$. In this case the nonlinear functional $\mathcal{F}_{h}(p)$ does not satisfy conditions for provable convergence for most nonlinear solvers. In this section we study the effect of the novel preconditioning approach for the cases of $n>2$ and $n<2$. These cases can be classified as smooth for $n>2$ and nonsmooth for $n<2$. As we can see in Table 1, all soils considered in the previous sections belong to the case of $n<2$, the same types of soils we consider for the nonsmooth case in this section. For the smooth case we choose three types of soils described as Silt Loam and Guelph Loam in [36] and as Sand in [34]. The parameters of van Genuchten and Mualem models and relative permeability curves and their derivatives as functions of $p_{c}$ are presented in Table 10 and Fig. 6. The 
absolute permeability and porosity of the layers are the same as in the previous sections, see Table 1. For this group of numerical experiments we consider the mesh shown in Fig. 3. Since the main difference between these two cases is the behavior around point $p=p_{\text {atm }}$ we raise the water table by setting boundary conditions on the bottom boundary of the domain to the pressure value which corresponds to a water table height of $15 \mathrm{~m}$ in the case of the hydrostatic pressure profile. This forces the critical point $p=p_{\text {atm }}$ to be within the domain. The rest of the boundary conditions stay the same as described in Section 4.2.

\begin{tabular}{c|c|c|c|c|c|c} 
& \multicolumn{4}{|c|}{ Nonsmooth } & \multicolumn{3}{|c}{ Smooth } \\
\cline { 2 - 7 } & $\alpha\left[\mathrm{Pa}^{-1}\right]$ & $m[-]$ & $n[-]$ & $\alpha\left[\mathrm{Pa}^{-1}\right]$ & $m[-]$ & $n[-]$ \\
\hline Top layer & $2.0674 \cdot 10^{-4}$ & 0.3006 & 1.43 & $3.5959 \cdot 10^{-4}$ & 0.6855 & 3.18 \\
Middle layer & $1.9467 \cdot 10^{-4}$ & 0.2294 & 1.30 & $5.1078 \cdot 10^{-4}$ & 0.8590 & 2.06 \\
Bottom layer & $2.0260 \cdot 10^{-3}$ & 0.2136 & 1.27 & $2.0431 \cdot 10^{-5}$ & 0.6377 & 2.03
\end{tabular}

Table 10: The van Genuchten parameters $\alpha, m$ and $n$ for the water retention models in different layers for nonsmooth and smooth relative permeability curves.

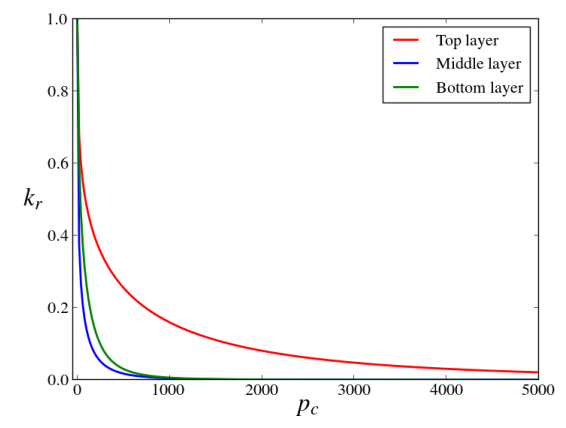

(a)

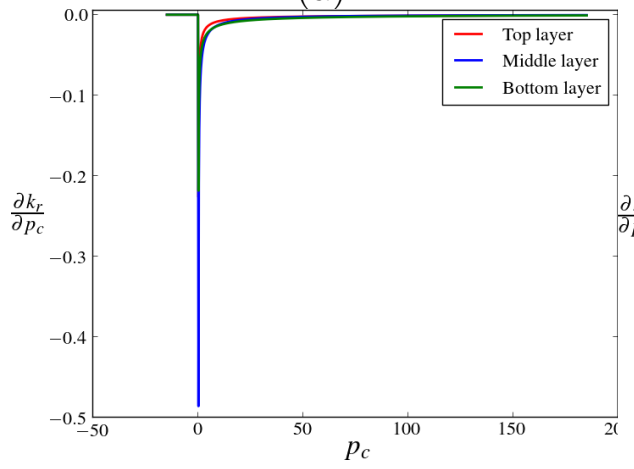

(c)

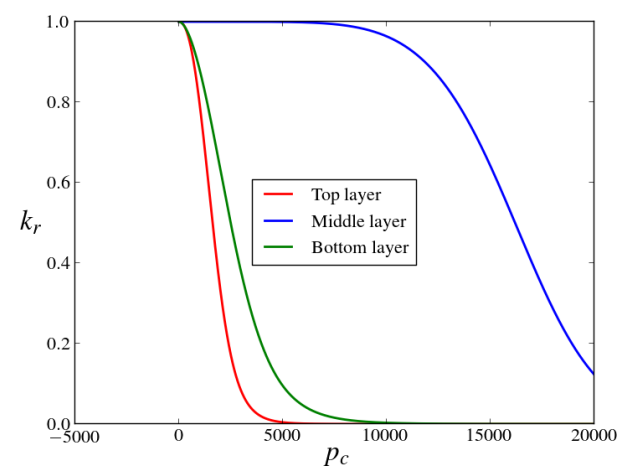

(b)

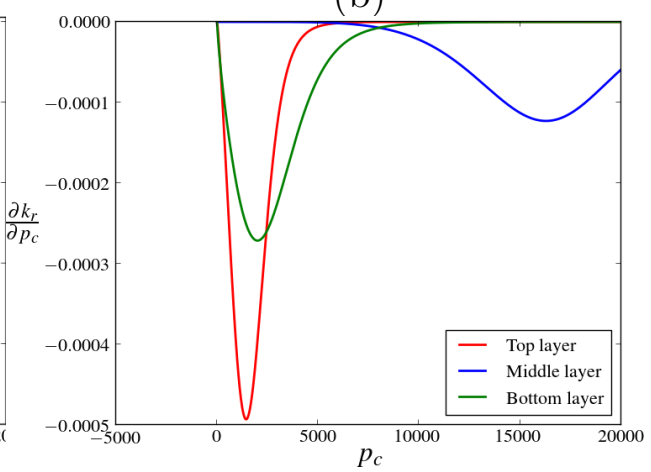

(d)

Figure 6: Relative permeability curve (a) and its derivative (c) for nonsmooth simulation. Relative permeability curve (b) and its derivative (d) for smooth simulation.

In this group of tests we run the transient simulation described in the previous section. The simulation starts from the steady-state solution precomputed for both sets of parameters. The transient phase starts at year 1956 and finishes at year 1959. The saturation profiles at different times are shown in Fig. 7. The media is more permeable in the smooth case so the water front reaches the water table faster and soils are less saturated. 
Both datasets presented in Tables 11-12 correspond to three year simulations, although the active phase (significant changes in solutions) of the smooth problem is much shorter than for the nonsmooth one. Therefore the speedup provided by the new preconditioning strategy only for the active phase is higher than presented in Tables 11-12.

As it can be expected, the nonsmooth problem is more challenging for nonlinear solvers, particularly for "discretization" preconditioner $\widehat{\mathcal{J}}_{h}^{(d)}$, see Tables 11-12. In the nonsmooth case preconditioner $\widehat{\mathcal{J}}_{h}^{(d)}$ does not provide convergence on most of time steps which leads to a significant reduction of a time step and an abort of the simulation. The new preconditioner $\widehat{\mathcal{J}}_{h}^{(l)}$ allow us to complete the simulation although it takes more nonlinear iterations and time steps in comparison with the smooth problem. In practice the nonsmoothness of the relative permeability curve is often rectified by a regularization procedure around the discontinuity point $p=p_{a t m}$. This restores a a continuous derivative of $k_{r}$ function and makes the problem more favorable for nonlinear solvers. It is important to note that in these tests we did not apply any regularization procedure.

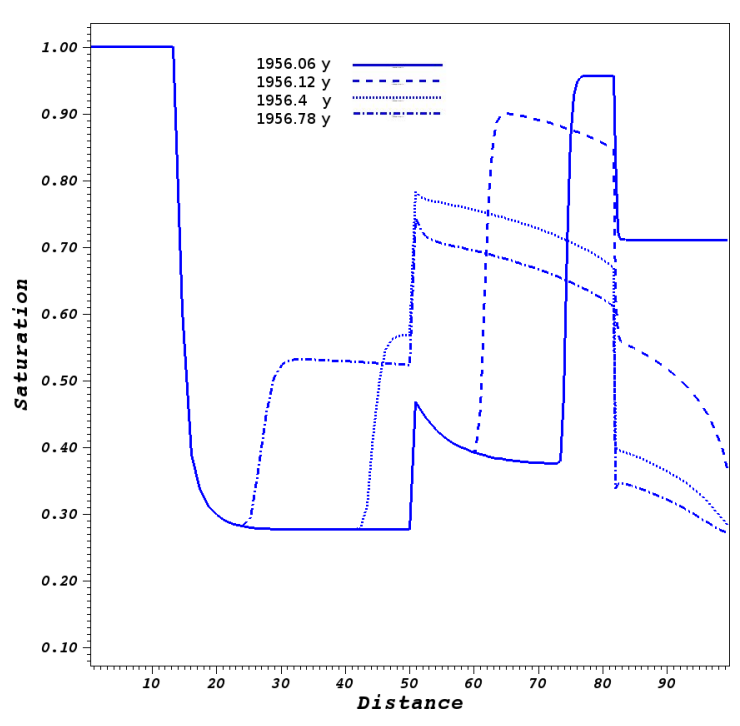

(a)

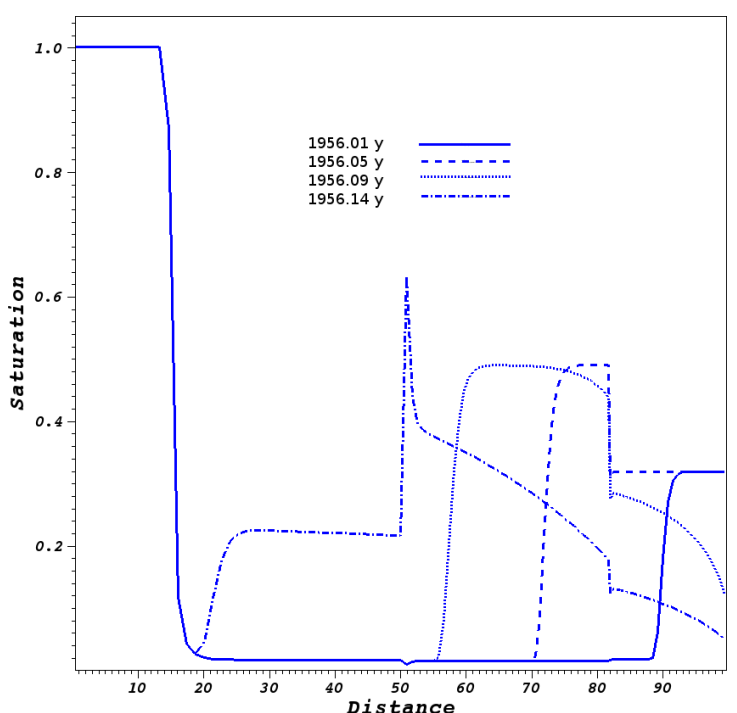

(b)

Figure 7: Saturation profiles at $x=100 \mathrm{~m}$ for different times for nonsmooth (a) and smooth (b) problems.

\section{Conclusion}

We described a new preconditioner for Richards' equation and analyzed numerically its impact on the convergence rate of three popular Jacobin-free nonlinear solvers, JFNK, NKA, and Picard. We considered challenging problems in subsurface hydrology (water infiltration into a partially saturated layered porous medium) that require non-orthogonal meshes and advanced discretization schemes. For all solvers, we observed consistent reduction (up to 40 times for some solution strategies and 3 times with respect to the state-of-art nonlinear solvers) of the the CPU time on fine meshes. The Picard solver with the new preconditioner was computationally more efficient than the JFNK and accelerate Picard solvers. The proposed strategy for Jacobian Free nonlinear solvers provides efficient and 


\begin{tabular}{c|c|c|c|c||c|c|c|c}
\multirow{2}{*}{} & \multicolumn{6}{|c||}{ \# successful time steps(\# failed time steps) } \\
\cline { 2 - 9 } & \multicolumn{4}{|c|}{ Nonsmooth } & \multicolumn{4}{c}{ Smooth } \\
\cline { 2 - 9 } & \multicolumn{2}{|c|}{ NKA } & \multicolumn{2}{c|}{ Picard } & \multicolumn{2}{c}{ NKA } & \multicolumn{2}{c}{ Picard } \\
\cline { 2 - 9 } & $\widehat{\mathcal{J}}_{h}^{(d)}$ & $\widehat{\mathcal{J}}_{h}^{(l)}$ & $\widehat{\mathcal{J}}_{h}^{(d)}$ & $\widehat{\mathcal{J}}_{h}^{(l)}$ & $\widehat{\mathcal{J}}_{h}^{(d)}$ & $\widehat{\mathcal{J}}_{h}^{(l)}$ & $\widehat{\mathcal{J}}_{h}^{(d)}$ & $\widehat{\mathcal{J}}_{h}^{(l)}$ \\
\hline mesh 64x64 & - & $173(25)$ & - & $143(31)$ & $368(11)$ & $165(8)$ & $1211(258)$ & $153(21)$ \\
\# nonlinear itr. & - & 1849 & - & 1515 & 3784 & 1528 & 12808 & 1264 \\
\hline mesh 128x128 & - & $309(80)$ & - & $244(26)$ & $541(1)$ & $250(15)$ & $1801(343)$ & $216(36)$ \\
\# nonlinear itr. & - & 3754 & - & 2394 & 5632 & 2543 & 19503 & 2003
\end{tabular}

Table 11: Transient flow simulation for the nonsmooth and smooth cases: the number of successful and failed time steps and the total number of nonlinear iterations. (-) means that the simulation cannot be completed due to a dramatic reduction of a time step.

\begin{tabular}{c|c|c|c|c||c|c|c|c}
\multirow{2}{*}{ mesh } & \multicolumn{9}{c|}{ CPU times $(\mathrm{sec})$} \\
\cline { 2 - 9 } & \multicolumn{3}{|c||}{ Nonsmooth } & \multicolumn{4}{c}{ Smooth } \\
\cline { 2 - 9 } & \multicolumn{2}{|c|}{ NKA } & \multicolumn{2}{c|}{ Picard } & \multicolumn{2}{c|}{ NKA } & \multicolumn{2}{c}{ Picard } \\
\cline { 2 - 9 } & $\widehat{\mathcal{J}}_{h}^{(d)}$ & $\widehat{\mathcal{J}}_{h}^{(l)}$ & $\widehat{\mathcal{J}}_{h}^{(d)}$ & $\widehat{\mathcal{J}}_{h}^{(l)}$ & $\widehat{\mathcal{J}}_{h}^{(d)}$ & $\widehat{\mathcal{J}}_{h}^{(l)}$ & $\widehat{\mathcal{J}}_{h}^{(d)}$ & $\widehat{\mathcal{J}}_{h}^{(l)}$ \\
\hline $64 \times 64$ & - & 225.5 & - & 188.3 & 321.9 & 169.5 & 1009.9 & 163.2 \\
$128 \times 128$ & - & 1779.7 & - & 1155.4 & 2059.2 & 1069.3 & 6784.5 & 883.0
\end{tabular}

Table 12: Computational cost of the transient flow simulation for the nonsmooth and smooth cases.

robust preconditioning approach which can be naturally combined with various advanced discretization methods to reproduce important features for multiphysics applications.

\section{Acknowledgement}

This work was carried out under the auspices of the National Nuclear Security Administration of the U.S. Department of Energy at Los Alamos National Laboratory under Contract No. DE-AC52-06NA25396. The authors acknowledge the support of the US Department of Energy Office of Science Advanced Scientific Computing Research (ASCR) Program in Applied Mathematics Research and the DOE Office of Environmental Management Advanced Simulation Capability for Environmental Management (ASCEM) Program.

The numerical experiments were performed with ASCEM's code Amanzi which is developed by a multi-institutional team spanning several DOE laboratories (http://ascemdoe.org/).

\section{References}

[1] Amanzi. https://software.lanl.gov/ascem/amanzi.

[2] D. Anderson. Iterative procedures for nonlinear integral equations. J. ACM, 12(4):547$560,1965$.

[3] B. Belfort, A. Younes, M. Fahs, and F. Lehmann. On equivalent hydraulic conductivity for oscillation-free solutions of Richards equation. Journal of Hydrology, 505:202-217, 2013. 
[4] L. Bergamaschi and M. Putti. Mixed finite elements and Newton-type linearizations for the solution of Richards' equation. Int. J. Numer. Meth. Eng., 45(8):1025-1046, 1999.

[5] C. G. Broyden. A class of methods for solving nonlinear simultaneous equations. Math. Comput., 19:577-593, 1965.

[6] M.T. Calef, E. D. Fichtl, J. S. Warsa, M. Berndt, and N. Carlson. Nonlinear krylov acceleration applied to a discrete ordinates formulation of the k-eigenvalue problem. Journal of Computational Physics, 238:188-209, 2013.

[7] N. Carlson and K. Miller. Design and application of a gradient-weighted moving finite element code, I: In one dimension. SIAM J. Sci. Comp., 19(3):728-765, 1998.

[8] N.N Carlson and K. Miller. Design and application of a gradient-weighted moving finite element code i: in one dimension. SIAM Journal on Scientific Computing, $19(3): 728-765,1998$.

[9] M. A. Celia and E. T. Bouloutas. A general mass-conservative numerical solution for the unsaturated flow equation. Water Resour. Res., 26(7):1483-1496, 1990.

[10] M.A. Celia, L.R. Ahuja, and G.F. Pinder. Orthogonal collocation and alternatingdirection procedures for unsaturated flow problems. Advances in Water Resources, 10:178-187, 1987.

[11] L. Chacón and D.A. Knoll. A 2D high- $\beta$ Hall MHD implicit nonlinear solver. J. Comput. Phys., 188(2):573-592, 2003.

[12] R.S. Dembo, S.C. Eisenstat, and T. Steihaug. Inexact Newton methods. SIAM J. Numer. Anal., 19(2):400-408, 1982.

[13] J. A. Dieudonné. Foundations of modern analysis. New York : Academic Press, 1960.

[14] J. Droniou, R. Eymard, T. Gallouet, and R. Herbin. A unified approach to mimetic finite difference, hybrid finite volume and mixed finite volume method. Math. Model. Methods Appl. Sci., 20(2):1-31, 2010.

[15] V. Eyert. A comparative study on methods for convergence acceleration of iterative vector sequences. J. Comput. Phys., 124(2):271-285, 1996.

[16] R. D. Falgout, J. E. Jones, and U. M. Yang. Numerical solution of partial differential equations on parallel computers., volume 51 of Lecture notes in computational science and engineering, chapter The design and implementation of hypre, a library of parallel high performance preconditioners., pages 267-294. Springer-Verlag, 2006.

[17] V. Gyrya, K. Lipnikov, G. Manzini, and D. Svyatskiy. M-adaptation in the mimetic finite difference method. Mathematical Models \& Methods In Applied Sciences, 24(8):1621-1663, 2014. 
[18] M.R. Hestenes and E.L. Stiefel. Methods of conjugate gradients for solving linear systems. J. Res. Natl. Bureau of Standards, Section B(49):409-436, 1952.

[19] J. E. Jones and C. S. Woodward. Preconditioning Newton-Krylov methods for variably saturated flow. In L. R. Bentley, J. F. Sykes, C. Brebbia, W. Gray, and G. F. Pinder, editors, Proceedings of the XIII International Conference on Computational Methods in Water Resources, pages 101-106, Calgary, Alberta, June 25-29, 2000.

[20] E. T. Karra, S. L. Painter, and P. C. Lichtner. Three-phase numerical model for subsurface hydrology in permafrost-affected regions (pflotran-ice v1.0). The Cryosphere, 8(5):1935-1950, 2014.

[21] C.T. Kelley. Iterative methods for linear and nonlinear equations. SIAM, Philadelphia, 1995.

[22] D. Knoll and D. Keyes. Jacobian-free Newton-Krylov methods: a survey of approaches and applications. J. Comput. Phys., 193(2):357-397, 2004.

[23] S. H. Lee, P. Jenny, and H. A. Tchelepi. A finite-volume method with hexahedral multiblock grids for modeling flow in porous media. Computational Geosciences, 6(34):353-379, 2002.

[24] K. Lipnikov, G. Manzini, J.D. Moulton, and M. Shashkov. The mimetic finite difference method for elliptic and parabolic problems with a staggered discretization of diffusion coefficient. J. Comp. Phys., 305:113-126, 2016.

[25] K. Lipnikov, D. Svyatskiy, and Y. Vassilevski. A monotone finite volume method for advection-diffusion equations on unstructured polygonal meshes. J. Comput. Phys., 229(11):4017-4032, 2010.

[26] K. Lipnikov, D. Svyatskiy, and Yu. Vassilevski. Minimal stencil finite volume scheme with the discrete maximum principle. Russian J. Numer. Anal. Math. Model., 27(4):369-385, 2012.

[27] P.A. Lott, H.F. Walker, C.S. Woodward, and U.M. Yang. An accelerated picard method for nonlinear systems related to variably saturated flow. Advances in Water Resources, 38:92-101, 2012.

[28] C.T. Miller, M.W. Farthing, C.E. Kees, and C.T. Kelley. Higher order, locally conservative temporal integration methods for modeling multiphase flow in porous media. In William G. Gray S. Majid Hassanizadeh, Ruud J. Schotting and George F. Pinder, editors, Computational Methods in Water ResourcesProceedings of the XIVth International Conference on Computational Methods in Water Resources (CMWR XIV), volume 47 of Developments in Water Science, pages 249-256. Elsevier, 2002.

[29] O. Misiats and K. Lipnikov. Second-order accurate monotone finite volume scheme for Richards' equation. J. Comp. Phys., 239:123-137, 2013.

[30] J. Nocedal and S. J. Wright. Numerical Optimization. New York : Springer, 2006. 
[31] Lichtner P.C., Hammond G.E., Lu C., Karra S., Bisht G., Andre B., Mills R. T., and Kumar J. "PFLOTRAN user manual". Technical report, 2013.

[32] M. Pernice and H.F. Walker. Nitsol: a Newton iterative solver for nonlinear system. SIAM Journal on Scientific and Statistical Computing, pages 302-318, 1998.

[33] L.A. Richards. Capillary conduction of liquids through porous mediums. Physics 1, 5:318-333, 1931.

[34] M. G. Schaap and F. J. Leij. Database-related accuracy and uncertainty of pedotransfer functions. Soil Science, 163(10):765-779, 1998.

[35] Michael D. Tocci, C.T. Kelley, and Cass T. Miller. Accurate and economical solution of the pressure-head form of richards' equation by the method of lines. Adv. Water Resour., 20(1):1-14, 1997.

[36] M. van Genuchten. A closed-form equation for predicting the hydraulic conductivity of unsaturated soils. Soil Sci. Soc. Am. J., 44:892-898, 1980.

[37] D. Vidović, M. Dotlić, B. Pokorni, M. Puzić, and M. Dimkic. Simulating unsaturated flow with a finite volume method. Water Research and Management, 4(1):23-30, 2014.

[38] H. F. Walker and P. Ni. Anderson acceleration for fixed-point iterations. SIAM Journal on Numerical Analysis, 49(4):1715-1735, 2011.

[39] M. White, D. Bacon, V. Freedman, M. Oostrom, M. Rockhold, S. White, and F. Zhang. Stomp Web page. http://stomp.pnnl.gov.

[40] A. Younes, M. Fahs, and B. Belfort. Monotonicity of the cell-centred triangular MPFA method for saturated and unsaturated flow in heterogeneous porous media. Journal of Hydrology, 504(11):132-141, 2013.

[41] J. Yue and G. Yuan. Picard-Newton iterative method with time step control for multimaterial non-equilibrium radiation diffusion problem. Commun. Comput. Phys., 10:844-866, 2011. 